\title{
SPATIO-TEMPORAL FILTERING OF THE EEG VIA NEURAL NETWORK BASED MULTIREFERENCE ADAPTIVE NOISE CANCELLING
}

\author{
Christopher J. James ${ }^{1 *}$, Student Member, IEEE, Martin T. Hagan ${ }^{4}$, Member, IEEE, \\ Richard D. Jones', Senior Member, IEEE, Philip J. Bones ${ }^{1}$, Senior Member, IEEE, and Grant J. Carroll ${ }^{3}$ \\ ${ }^{1}$ Department of Electrical and Electronic Engineering, University of Canterbury \\ ${ }^{2}$ Department of Medical Physics \& Bioengineering, Christchurch Hospital \\ ${ }^{3}$ Department of Neurology, Christchurch Hospital \\ Christchurch, New Zealand \\ ${ }^{4}$ School of Electrical and Computer Engineering, Oklahoma State University, USA
}

\begin{abstract}
A system is proposed which enhances transient nonstationarities and, in particular, epileptiform discharges in the EEG. It is based around the technique of multireference adaptive noise cancelling (MRANC) which attenuates the background EEG on a primary channel by using spatial and temporal information from adjacent channels in the multichannel EEG recording. This process has been implemented by means of a 3-layer perceptron artificial neural network trained by a backpropagation algorithm.

System performance was measured as the percentage increase in signal-to-noise ratio (SNR) of predetermined epileptiform discharges in recorded EEG segments. The results obtained show that, due to the nonlinear nature of the artificial neural network, the improvement in SNR is significant when compared to the performance of MRANC utilising a linear model.

MRANC is proposed as the first stage of a neural network based multi-stage system to detect epileptiform discharges in the interictal EEG for the diagnosis of epilepsy.
\end{abstract}

\section{INTRODUCTION}

$\mathrm{T}$ HE electroencephalogram (EEG) can be considered as consisting of an underlying background process, assumed to be stationary and ergodic, onto which are superimposed transient non-stationarities (TNSs) such as spike and sharpwaves (SSWs), electrode 'pop', eye-blinks, and muscle artifacts. The detection of SSWs in the EEG is of particular importance in the diagnosis of epilepsy. Several approaches have been made towards automating the detection of SSWs with widely varying performances, e.g., [1-3].

The method described here comprises the first stage of an ANN-based system designed to detect SSWs in the interictal EEG. It essentially processes the EEG by attenuating the background EEG, thus primarily leaving only TNSs which are then classified as SSW or non-SSW by a second stage which is not described here.

The system makes use of multireference adaptive noise cancelling (MRANC), as described by Widrow et al. [4]. The

\footnotetext{
* e-mail: jamescj@elec.canterbury.ac.nz
}

use of multilayer ANNs to implement the MRANC filters provides the opportunity to model the EEG spatial distribution as nonlinear, leading to improved performance over the linear case.

\section{Methods}

\section{A. Data collection}

Sixteen channels of scalp EEG were recorded simultaneously both for referential and bipolar montages. The amplified EEG was bandpass filtered between 0.5 and $70 \mathrm{~Hz}$, sampled at $200 \mathrm{~Hz}$ and digitized to 12 bits. All data were stored for later off-line processing.

Single segments of bipolar EEG, each containing $10 \mathrm{SSW}$ (as identified by an electroencephalographer), were chosen from the epileptiform EEGs of six patients. So as to test the system on a range of different SSWs, the EEGs chosen included both focal SSWS and generalised SSWS.

\section{B. Performance index}

To measure the performance of the system, the signal-tonoise ratio (SNR) was defined as the ratio of the peak-to-peak value of the SSW to the root-mean-square value of the background EEG for 30 samples $(150 \mathrm{~ms})$ on either side of the SSW, excluding the SSW itself.

\section{Multireference adaptive noise cancelling}

As we are interested in detecting TNSs in the EEG, and bearing in mind that the interictal EEG can be considered as consisting of a separate background process $N(t)$ onto which are superimposed TNSs $S(t)$, we model the signal recorded at each scalp electrode as the weighted sum of $N(t)$ and $S(t)$.

To perform MRANC, separate reference inputs are required which yield samples of the noise sources only. The filtered reference inputs are subtracted from the primary input carrying the signal and noise, leaving the original signal free of noise (Fig. 1). 


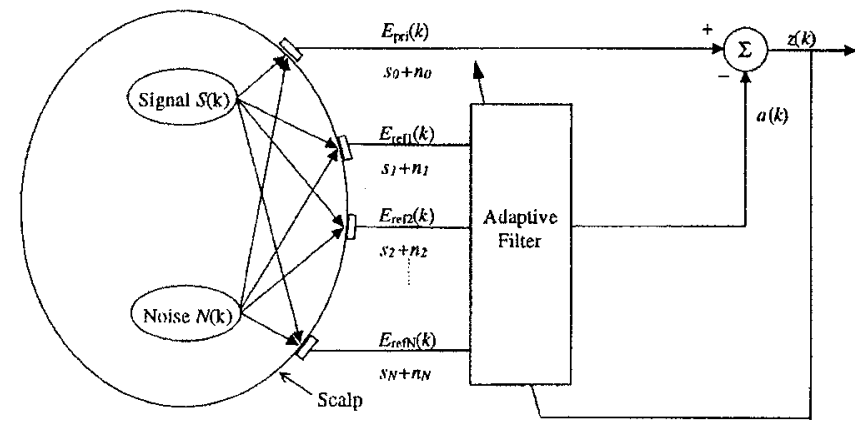

Fig. 1. Multireference adaptive noise canceller

Sometimes the reference inputs may contain some signal components which arc correlated to the signal at the primary input. This 'cross-talk' will cause some cancellation of the signal in the primary input.

MRANC was implemented by a 3-layer ANN where each neuron in the hidden layer contains a log-sigmoidal nonlinear activation function and the single output neuron has a linear activation function. The backpropagation algorithm was used to train the ANN. For comparison, MRANC was also implemented using the linear least mean squared (LMS) algorithm.

The channel containing the highest amplitude SSWs, generally being closest to the epileptogenic focus, was made the primary channel. The reference channels were then grouped as follows: group A comprised of the 3 channels closest to the primary channel, group B the 4 channels furthest from the primary channel and group $C$ all channels other than the primary channel. MRANC was then performed on each segment via each of the reference groups. The SNR of the SSW at the output of the MRANC filter was recorded and the percentage increase in SNR calculated.
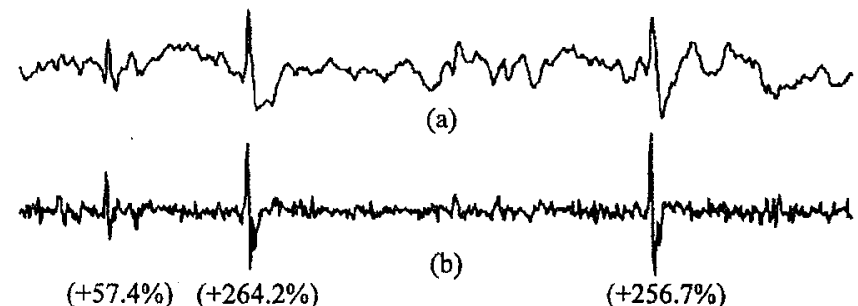

Fig. 2. Results of MRANC on three SSWs. (a) The primary signal and (b) the nonlinear MRANC filter output ( 2 neurons in the hidden layer, closest channels as reference channels). The \% incrcase in SNR is indicated by the values in brackets.

\section{Results}

MRANC achieved an increase in the average SNR of SSWs in all patients for both linear and nonlinear configurations. However, in nearly all SSWs tested, the nonlinear MRANC configuration resulted in a significant improvement in performance over the linear configuration. Overall, increasing the number of neurons in the hidden layer above 10 resulted in no significant improvement in performance. Furthermore, performance increased slightly as more channels were included in the reference groups. Fig. 2 depicts an example of three SSWs in the EEG segment of patient \#1. The average percentage increase in SNR over the six patients for 10 neurons in the hidden layer was $121 \%$, compared to an average of $76 \%$ for the linear case.

\section{DISCUSSION}

We contend is that SSW classification can be made considerably more accurate in terms of both sensitivity and selectivity by the prior attenuation of the background EEG. Implementing MRANC by means of an ANN allows the process to be modelled as nonlinear which has been shown to yield considerably better results than its linear counterpart. The adaptive nature of the MRANC filter also allows for variations in the background EEGs of different patients to be optimally accommodated. By designating all channels other than the primary channel as reference channels (i.e., reference group $\mathrm{C}$ ), the need for arbitrary selection of reference groups is removed.

In conclusion, MRANC can considerably enhance the presence of both focal and generalised activity in the EEG and the use of nonlinear ANNs in the application of MRANC improves the effectiveness of the process. By enhancement of TNSs, in particular SSWs, MRANC can become an important first stage in the detection of epileptiform activity in the interictal EEG.

\section{REFERENCES}

[1] J. Gotman, "Automatic recognition of interictal spikes", in Long term monitoring in epilepsy EEG supplement No. 37, J. Gotman, J.R. Ives, and P. Gloor, Eds., pp. 99-114. Elsevier, 1985.

[2] A.A. Dingle, R.D. Jones, G.J. Carroll and W.R. Fright, "A multistage system to detect epileptiform activity in the EEG", IEEE Trans. Biomed. Eng., vol. 40, pp. 1260-1268, 1993.

[3] W.R.S. Webber, B. Litt, K. Wilson and R.P. Lesser, "Practical detection of epileptiform discharges (EDs) in the EEG using an artificial neural network: a comparison of raw and parameterized EEG data", Electroenceph. Clin. Neurophysiol., vol. 91, pp. 194204, 1994.

[4] B. Widrow, J.R. Glover, J.M. McCool, J. Kaunitz, C.S. Williams, R.H. Hearn, J.R. Zeidler, E. Dong, and R.C. Goodlin, "Adaptive noise cancelling: principles and applications", Proc. IEEE, vol. 63, pp. 1692-1716, 1975. 